Check for updates

Cite this: RSC Adv., 2018, 8, 37348

Received 11th September 2018 Accepted 31st October 2018

DOI: $10.1039 / c 8 r a 07547 f$

rsc.li/rsc-advances

\section{Rare earth complexes using azobenzene- containing poly(aryl ether)s with different absorption wavelengths as macromolecular ligands: synthesis, characterization, fluorescence properties and fabrication of fluorescent holographic micropatterns}

\begin{abstract}
Yuxuan Zhang ${ }^{a}$ and Shanyong Chen (iD *b
In this paper, two novel azobenzene-containing poly(aryl ether)s with different absorption wavelengths were synthesized via Ullmann coupling and Sonogashira coupling, respectively. The obtained polymers were characterized and evaluated by elemental analysis, IR, ${ }^{1} \mathrm{H} N M R$, UV-vis, DSC and TGA. Rare earth complexes were prepared by using the two novel azobenzene-containing poly(aryl ether)s as macromolecular ligands. The obtained rare earth complexes were characterized by elemental analysis, IR and WAXD. The influence of the absorption wavelength of azobenzene chromophores on the fluorescent properties was investigated. The polymer whose absorption wavelength was far from the excitation wavelengths of the rare earth complexes showed a much larger fluorescence intensity. By exposing the films of the rare earth complexes to two interference laser beams, SRGs can be formed on the films and can also be detected by fluorescence microscopy measurement.
\end{abstract}

\section{Introduction}

Azobenzene-containing polymers (azo-polymers) have attracted much attention due to their potential application in optical data storage, photochemical switches, photodeformation materials and nonlinear optical materials. ${ }^{1-8}$ One of the attractive phenomena is that surface relief gratings (SRGs) can be formed directly on the films of azo-polymers by exposing the films to two interfering laser beams due to the reversible photoisomerization and photoinduced anisotropy of the azobenzene groups., ${ }^{9,10}$ The SRGs are stable below the glass transition temperatures $\left(T_{\mathrm{g}} \mathrm{s}\right)$ of the polymers and can be removed by heating the polymers above their $T_{\mathrm{g}} \mathrm{s}$ or can be erased optically. Therefore, introducing azobenzene chromophores into some high- $T_{\mathrm{g}}$ aromatic polymers, such as polyimides and poly(aryl ether)s, can improve the storage stability of SRGs based on the azobenzene-containing polymers. ${ }^{\mathbf{1 1 - 1 5}}$

In recent years, a facile method for fabricating fluorescent and rewritable micropatterns by the combination of the holographic technique and fluorescence technique was demonstrated. ${ }^{\mathbf{1 6 - 1 8}}$ The method could be realized through the combination of fluorescent materials and azobenzene-containing materials. The

${ }^{a}$ College of Chemistry, Liaoning University, Shenyang, 110036, China

${ }^{b}$ Research Institute for New Materials Technology, Chongqing University of Arts and Sciences, Yongchuan 402160, China studies provided a convenient method to fabricate patterned organic light-emitting devices and opened up a new way of recording and detecting optical information. In our previous work, a series of novel materials for fabricating fluorescent holographic micropatterns based on the rare earth coordination of poly(aryl ether)s with different azobenzene chromophores contents was demonstrated. ${ }^{19}$ With the introduction of azobenzene chromophores and the increase of azobenzene chromophore content, the fluorescence intensity of the rare earth complexes decreased dramatically. The reason is that the excitation wavelengths of the rare earth complexes overlapped with the absorption wavelength of the azobenzene chromophores, and the fluorescence intensity of the rare earth complexes was weakened by the azobenzene chromophores. To essentially prevent such energy competition between azobenzene chromophores and rare earth complexes, the structure of the azobenzene chromophores should be carefully designed to make the absorption wavelengths of the azobenzene chromophores far away from the excitation wavelengths of the rare earth complexes.

In this work, novel poly(aryl ether)s with carboxyl side groups and bromine side groups (Br-CPAE) were synthesized. Based on the novel polymer Br-CPAE, a novel azobenzene-containing poly(aryl ether) whose absorption wavelength was far away from the excitation wavelengths of rare earth complexes was synthesized via Sonogashira coupling. For comparison, a novel azobenzene-containing poly(aryl ether) whose absorption 
wavelength overlapped with the excitation wavelengths of rare earth complexes was also synthesized via Ullmann coupling. The rare earth complexes were prepared by using the three novel poly(aryl ether)s as macromolecular ligands. The influence of the absorption wavelength of azobenzene chromophores on the fluorescent properties was investigated. By exposing the films of the rare earth complexes to two interference laser beams, SRGs could be formed on the films. Under the excitation, red fluorescent patterns of the SRGs could be detected by fluorescence microscopy measurement.

\section{Materials}

\subsection{Materials}

4-Fluorophenyl sulfone, $\mathrm{Ce}_{2} \mathrm{CO}_{3}$, $\mathrm{CuCl}$, CuI, triethylamine, 3ethynylaniline and $\mathrm{N}, \mathrm{N}$-dimethylaniline were purchased from Aladdin. Phenolphthalin was purchased from TCI. 2,2,6,6Tetramethyl-3,5-heptanedione (TMHD) was purchased from Aldrich. 2-(4-Bromophenyl)hydroquinone and 4-((4-methoxyphenyl)diazenyl)phenol (MDP) were synthesized according to the literature, ${ }^{20,21}$ respectively. All of the other reagents were purchased from commercial sources.

\subsection{Measurements}

${ }^{1} \mathrm{H}$ NMR spectra were recorded on a Mercury-Vx300-NMR instrument using DMSO- $\mathrm{d}_{6}$ as the solvent. UV-visible absorption spectra were recorded on a PerkinElmer Lambda 35 UV-vis spectrophotometer at room temperature. Elemental analysis was conducted on a Perkin-Elmer 2400 Elemental analyser. The elemental analysis of the content of $\mathrm{Eu}^{3+}$ was conducted using an Optima 3300DV inductively coupled plasma system. FT-IR spectra ( $\mathrm{KBr}$ pellet) were recorded on a Perkin Elmer Spectrum One FT-IR spectrophotometer. Gel permeation chromatography was carried out using a Waters 410 instrument with polystyrene as the calibration standard. Wide-angle X-ray diffraction (WAXD) measurements were carried out on a Shimadzu XRD-7000 X-ray diffractometer with a $\mathrm{Cu} \mathrm{K} \alpha$ radiation source. The powder sample was multiplied to increase the intensity in the $2 \theta$ range of $5-60^{\circ}$. Glass transition temperatures $\left(T_{\mathrm{g}} \mathrm{s}\right)$ were determined by a TA DSC-Q100 instrument under nitrogen atmosphere at a heating rate of $10{ }^{\circ} \mathrm{C} \mathrm{min}^{-1}$. Thermogravimetric analysis was performed on a TA SDT-Q600 TGA analyser under nitrogen atmosphere at a heating rate of $10{ }^{\circ} \mathrm{C} \mathrm{min}{ }^{-1}$. The fluorescence emission spectra of rare earth complexes were measured on an Edinburgh FS900 fluorescence spectrophotometer.

The experimental setups for SRGs formation have been described by our groups previously, ${ }^{14}$ and only several details are given here. The SRGs were optically inscribed on the spincoated films with two interfering laser beams. Polarized Nd:YAG nanosecond pulsed $355 \mathrm{~nm}$ laser beams with a pulse duration of $10 \mathrm{~ns}$ were utilized as the recording light source. The surface topology of the SRG films was observed using a Nanoscope atomic force microscope (AFM) in tapping mode. The fluorescence microscopy images of the SRGs were obtained on an Olympus BX51 Reflected Fluorescence System.

\subsection{Synthesis}

2.3.1. Synthesis of 4-((3-ethynylphenyl)diazenyl)- $N, N$ dimethylaniline (monomer 1, Scheme 1). Monomer 1 was synthesized by a diazotization reaction followed by coupling with phenol. Water $(50 \mathrm{~mL})$ and 3-ethynylaniline $(0.05 \mathrm{~mol})$ were stirred with a mechanical stirrer in a $500 \mathrm{~mL}$ beaker. Hydrochloric acid $(0.1 \mathrm{~mol}, 8.4 \mathrm{~mL})$ was added dropwise into the stirred mixture. The solution was cooled to $0-5{ }^{\circ} \mathrm{C}$ and then a water solution of sodium nitrite $(0.05 \mathrm{~mol})$ was added dropwise. The mixture solution was stirred for $30 \mathrm{~min}$ at $0-5{ }^{\circ} \mathrm{C}$. The resulting solution was filtered and added dropwise into a solution of $\mathrm{CH}_{3} \mathrm{COONa}(0.4 \mathrm{~mol})$ and $N, N$-dimethylaniline $(0.05$ mol). The reaction mixture was stirred at $0-5{ }^{\circ} \mathrm{C}$ for approximately $1 \mathrm{~h}$ and then at room temperature for $3 \mathrm{~h}$. The precipitate was collected by filtration and washed with water several times. The precipitate was dried at $60{ }^{\circ} \mathrm{C}$ in vacuum and then recrystallized from ethanol to obtain a yellow powder $(63 \%$ yield). ${ }^{1} \mathrm{H}$ NMR (DMSO- $\left.\mathrm{d}_{6}, \delta, \mathrm{ppm}\right):$ 7.80-7.82 (m, 4H), 7.53-7.55 $(\mathrm{m}, 2 \mathrm{H}), 6.84(\mathrm{~d}, 2 \mathrm{H}), 4.29(\mathrm{~s}, 1 \mathrm{H}), 3.07(\mathrm{~s}, 6 \mathrm{H})$.

2.3.2. Synthesis of poly(ether sulfone)s with bromine groups and carboxyl groups (Br-CPAE, Scheme 2). Phenolphthalin (0.008 mol), 2-(4-bromophenyl)hydroquinone $(0.002$ mol), 4,4'-dichlorodiphenyl sulfone $(0.01 \mathrm{~mol}), \mathrm{K}_{2} \mathrm{CO}_{3}(0.015$ $\mathrm{mol})$, DMSO $(20 \mathrm{~mL})$ and toluene $(8 \mathrm{~mL})$ were put into a threenecked flask. After dehydration and removal of toluene, the reaction mixture was heated at $160{ }^{\circ} \mathrm{C}$ for $6 \mathrm{~h}$ under nitrogen. After being slowly poured into aqueous $\mathrm{HCl}(800 \mathrm{~mL}, 4 \mathrm{~mol})$ under stirring, the precipitate was collected and washed with hot deionized water and ethanol several times. The obtained polymer powder was dried at $100^{\circ} \mathrm{C}$ under vacuum for $24 \mathrm{~h}$ and Br-CPAE was obtained as a white powder.

2.3.3. Synthesis of Azo-CPAE-1 (Scheme 3). Azo-CPAE-1 was synthesized via Ullmann coupling between Br-CPAE and MDP as follows. Br-CPAE $(2 \mathrm{mmol}), \mathrm{MDP}(1 \mathrm{mmol})$, TMHD $(0.08$ $\mathrm{mmol}), \mathrm{CuCl}(0.08 \mathrm{mmol})$ and $\mathrm{Cs}_{2} \mathrm{CO}_{3}(0.88 \mathrm{mmol})$ were

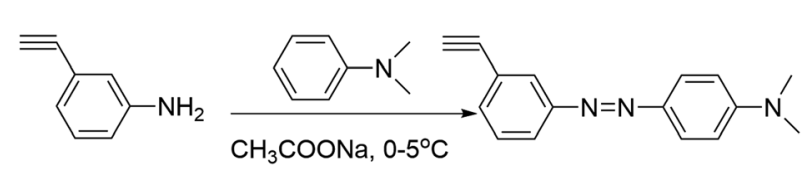

Scheme 1 Synthetic route of monomer 1.

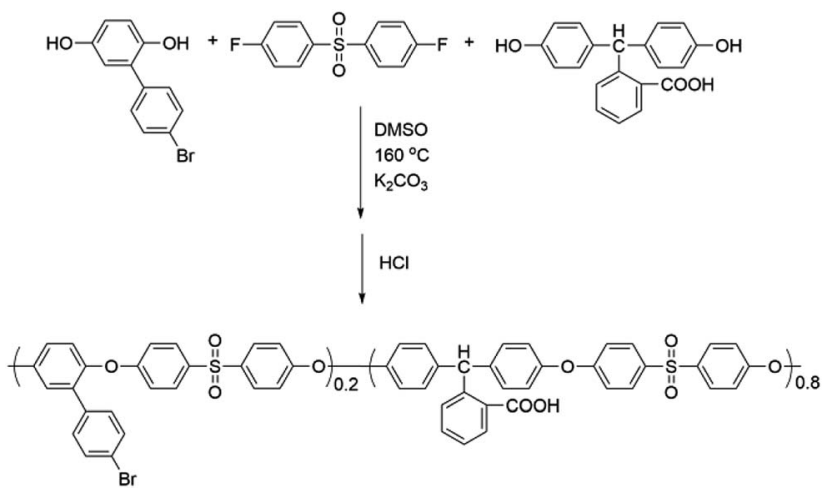

Scheme 2 Synthetic route of $\mathrm{Br}$-CPAE. 
dissolved in anhydrous DMAc. The mixture was degassed and filled with nitrogen, and then heated at $120{ }^{\circ} \mathrm{C}$ under nitrogen for $12 \mathrm{~h}$. After being slowly poured into aqueous $\mathrm{HCl}(200 \mathrm{~mL}, 1$ mol), the precipitate was collected and washed with hot deionized water and ethanol several times in sequence. The resulting polymer was dried at $100{ }^{\circ} \mathrm{C}$ under vacuum for $24 \mathrm{~h}$ and Azo-CPAE-1 was obtained as an orange-yellow powder.

2.3.4. Synthesis of Azo-CPAE-2 (Scheme 4). Azo-CPAE-2 was synthesized via Sonogashira coupling between Br-CPAE and monomer 1 as follows. Br-CPAE (2 mmol), bis(triphenylphosphine)dichloropalladium (0.04 mmol), CuI (0.04 $\mathrm{mmol})$, monomer 1 ( $1 \mathrm{mmol})$ and triethylamine $(0.84 \mathrm{mmol})$ were dissolved in anhydrous DMAc. The mixture was degassed and filled with nitrogen, and then heated at $100{ }^{\circ} \mathrm{C}$ under nitrogen for $12 \mathrm{~h}$. After being slowly poured into aqueous $\mathrm{HCl}$ (200 mL, $1 \mathrm{~mol}$ ), the precipitate was collected and washed with hot deionized water and ethanol several times in sequence. The resulting polymer was dried at $100{ }^{\circ} \mathrm{C}$ under vacuum for $24 \mathrm{~h}$ and Azo-CPAE- 2 was obtained as an orange-red powder.

2.3.5. Synthesis of rare earth coordination polymers (Scheme 5). The preparation procedure of the rare earth coordination polymers was synthesized according to the literature. ${ }^{19,22}$ As shown in Scheme 5, the synthesis route for the rare earth complex of $\mathrm{Br}-\mathrm{CPAE}\left(\mathrm{Eu}^{3+}\right.$-Br-CPAE) was described for example as follows: Br-CPAE (0.002 mol) was dissolved in $10 \mathrm{~mL}$ of dried DMF. Then, a solution of $\mathrm{Eu}\left(\mathrm{NO}_{3}\right)_{3}(0.0016 \mathrm{~mol})$ and Phen $(0.0048 \mathrm{~mol})$ in $10 \mathrm{~mL}$ of dried DMF was added. After stirring for $2 \mathrm{~h}$, the solution was slowly poured into $200 \mathrm{~mL}$. The

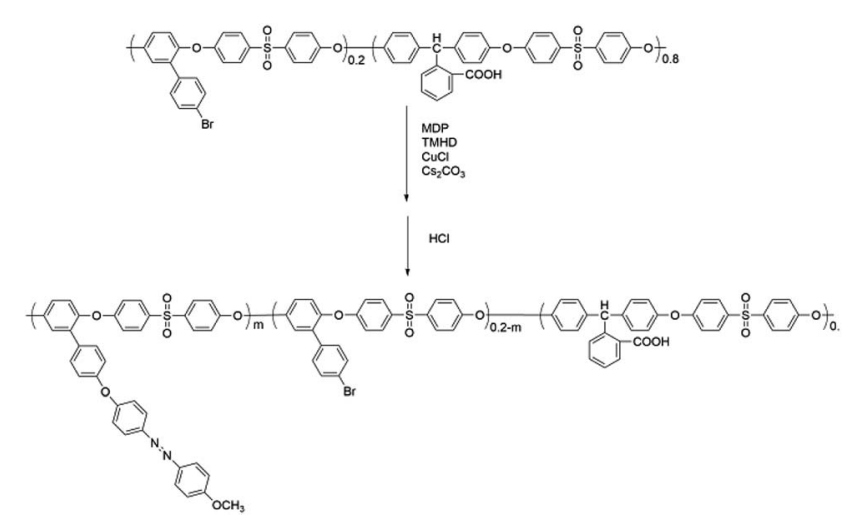

Scheme 3 Synthesis routes to Azo-CPAE-1.

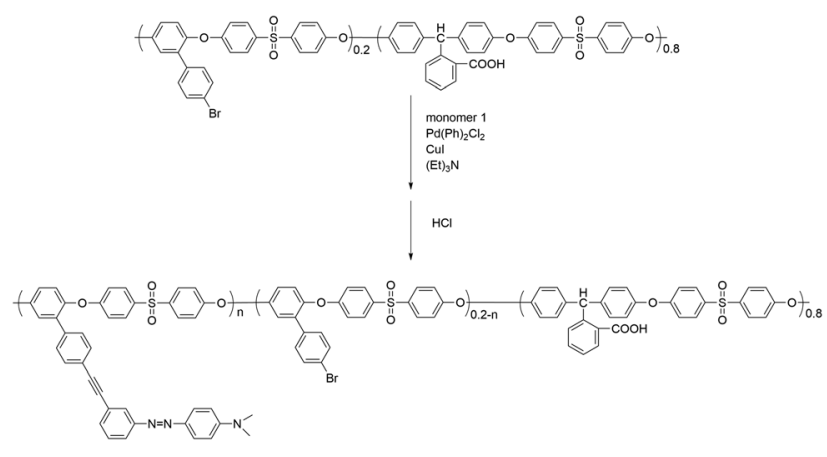

Scheme 4 Synthesis routes to Azo-CPAE-2.



Scheme 5 Synthesis routes to $\mathrm{Eu}^{3+}-\mathrm{Br}-\mathrm{CPAE}$.

precipitate was washed with ethanol several times, and then dried at $80{ }^{\circ} \mathrm{C}$ under vacuum for $24 \mathrm{~h}$. As demonstrated in other poly(aryl ether)s, ${ }^{22}$ the rare earth coordination polymer might own a stable eight-fold coordination structure. The possible molecular formula of the rare earth coordination polymer is shown in Scheme 5. The preparation procedures and structures of the rare earth complexes of Azo-CPAE-1 and Azo-CPAE-2 $\left(\mathrm{Eu}^{3+}\right.$-Azo-CPAE-1 and $\mathrm{Eu}^{3+}$-Azo-CPAE-2) were similar to those of $\mathrm{Eu}^{3+}$-Br-CPAE.

To compare with the rare earth coordination polymer, a small molecule rare earth complex $\mathrm{Eu}^{3+}$-Phen was synthesized as follows: 1,10-phenanthroline $(0.006 \mathrm{~mol})$ was dissolved in $6 \mathrm{~mL}$ of dried DMF. Then, a solution of $\mathrm{Eu}\left(\mathrm{NO}_{3}\right)_{3}(0.002 \mathrm{~mol})$ in $6 \mathrm{~mL}$ of dried DMF was added. The mixture was stirred for $3 \mathrm{~h}$. After being slowly poured into $50 \mathrm{~mL}$ of ethanol, the precipitate was collected and washed with ethanol several times. The product was dried at $80{ }^{\circ} \mathrm{C}$ under vacuum for $24 \mathrm{~h}$.

2.3.6. Preparation of the films of rare earth coordination polymers for SRGs. The films of the rare earth coordination polymers for SRGs were prepared by the following procedure. The rare earth coordination polymers were prepared from the polymers, $\mathrm{Eu}^{3+}$ ion and Phen at a molar ratio of $1: 0.8: 2.4$, which were dissolved in dried DMF (10 wt $\%)$. After stirring for $2 \mathrm{~h}$, the solution was filtered through $0.45 \mu \mathrm{m}$ syringe filter membranes. To fabricate SRGs, films were obtained via spincoating the solution onto clean glass substrates. The thickness was controlled to be approximately $1.0 \mu \mathrm{m}$ by adjusting the spinning rate. After drying under vacuum for $48 \mathrm{~h}$ to drive off the residual solvent, the films were obtained.

\section{Results and discussion}

\subsection{Synthesis and characterization}

We designed a new monomer containing the azobenzene group and the synthetic route of the monomer is illustrated in Scheme 1. The structure of monomer 1 was confirmed by ${ }^{1} \mathrm{H}$ NMR, as shown in Fig. 1. All of the signals are in good agreement with the expected structure.

Two novel azobenzene-containing poly(aryl ether)s (AzoCPAE-1 and Azo-CPAE-2) with different absorption 


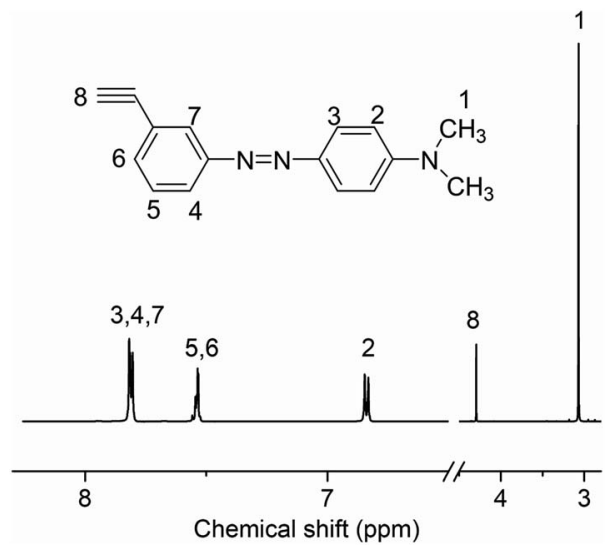

Fig. $1{ }^{1} \mathrm{H}$ NMR spectrum of monomer 1 in DMSO- $d_{6}$.

Table 1 Properties of the polymers

\begin{tabular}{lclc}
\hline Polymer & Grafting ratio\% & $M_{\mathrm{n}}$ & $M_{\mathrm{w}} / M_{\mathrm{n}}$ \\
\hline Br-CPAE & 0 & $1.1 \times 10^{4}$ & 1.81 \\
Azo-CPAE-1 & 87 & $1.0 \times 10^{4}$ & 2.12 \\
Azo-CPAE-2 & 93 & $1.2 \times 10^{4}$ & 1.94 \\
\hline
\end{tabular}

wavelengths were synthesized via Ullmann coupling and Sonogashira coupling, respectively. From Table 1, it could be seen that all the polymers had number average molecular weights above $1 \times 10^{4} \mathrm{~g} \mathrm{~mol}^{-1}$. Due to the high alkalinity of $\mathrm{Cs}_{2} \mathrm{CO}_{3}, \mathrm{Cs}_{2} \mathrm{CO}_{3}$ could degrade the main chain of Br-CPAE. Thus the $M_{\mathrm{n}}$ of Azo-CPAE-1 was lower than that of Br-CPAE. All the polymers showed good solubility in common organic solvents such as tetrahydrofuran, dimethylacetamide, $N, N$-dimethylformamide, $N$-methyl-2-pyrrolidone and cyclohexanone.

The chemical structures of the polymers were confirmed by elemental analysis, IR, ${ }^{1} \mathrm{H}$ NMR and UV-vis spectra. As shown in Table 2, on the basis of elemental analysis, the molar percentage of azobenzene chromophores in Azo-CPAE-1 and Azo-CPAE-2 could be calculated. If all of the aryl bromide reacted, it represented a $100 \%$ grafting ratio. The grafting ratios of Azo-CPAE-1 and Azo-CPAE-2 were $93 \%$ and $87 \%$, respectively. The IR spectra of the polymers are shown in Fig. 2. It could be observed that the IR spectra of the polymers showed characteristic absorption bands of carboxyl groups at $1718 \mathrm{~cm}^{-1}$, sulfone groups at $1295 \mathrm{~cm}^{-1}$ and aryl ether groups at $1238 \mathrm{~cm}^{-1}$, indicating that the polymers were successfully prepared. Fig. 3 shows the typical ${ }^{1} \mathrm{H}$ NMR spectra of Br-CPAE in DMSO- $\mathrm{d}_{6}$. All of the signals are in good agreement with the expected structure. Fig. 4 shows the typical ${ }^{1} \mathrm{H}$ NMR spectra of all the polymers in DMSO$\mathrm{d}_{6}$. Compared with the ${ }^{1} \mathrm{H}$ NMR spectra of Br-CPAE, the ${ }^{1} \mathrm{H}$ NMR spectra of Azo-CPAE-1 showed new peaks at approximately

Table 2 Elemental analysis of the polymers

\begin{tabular}{llll}
\hline Polymer & $\mathrm{C}$ & $\mathrm{H}$ & $\mathrm{N}$ \\
\hline Br-CPAE & 69.68 & 3.95 & \\
Azo-CPAE-1 & 71.43 & 4.13 & 0.89 \\
Azo-CPAE-2 & 72.25 & 4.23 & 1.41
\end{tabular}

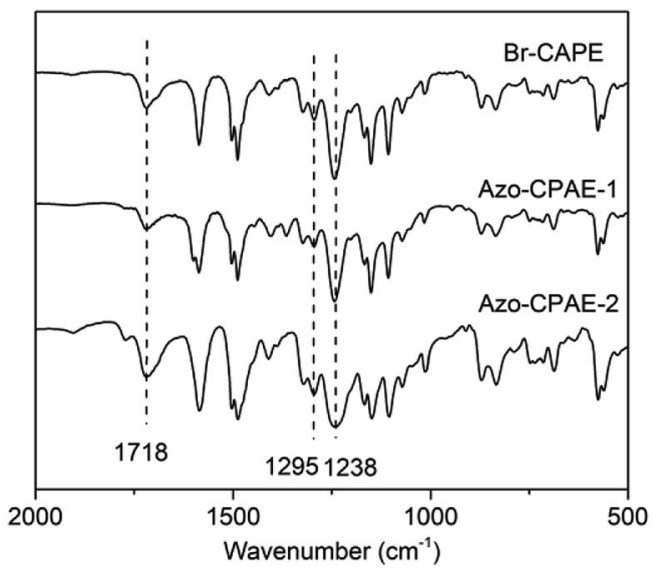

Fig. 2 IR spectra of the polymers.

$3.85 \mathrm{ppm}$ corresponding to the chemical shift of hydrogen in the $-\mathrm{OCH}_{3}$ group. Similarly, compared with the ${ }^{1} \mathrm{H}$ NMR spectra of Br-CPAE, the ${ }^{1} \mathrm{H}$ NMR spectra of Azo-CPAE-2 showed new peaks at approximately $3.01 \mathrm{ppm}$ corresponding to the chemical shift of hydrogen in the $-\mathrm{N}\left(\mathrm{CH}_{3}\right)_{2}$ groups. The UV-vis spectra of the polymers in DMF solution are shown in Fig. 5. The characteristic absorption bands at approximately $353 \mathrm{~nm}$ and $426 \mathrm{~nm}$ could be observed in the spectra of Azo-CPAE-1 and Azo-CPAE-2, respectively, corresponding to $\pi-\pi^{*}$ transitions resulting from the intramolecular charge transfer of the azobenzene chromophores. ${ }^{23}$ Due to the larger conjugate length, the absorption wavelength of azobenzene chromophores in Azo-CPAE-2 was larger than the absorption wavelength of azobenzene chromophores in Azo-CPAE-1.

The chemical structures of the rare earth coordination polymers could be confirmed by IR, WAXD and elemental analysis. For example, Fig. 6 presents the comparison of the IR spectra of DMF, Phen, Azo-CPAE-2 and the coordination polymer $\mathrm{Eu}^{3+}$-Azo-CPAE-2. From the IR spectra of DMF and $\mathrm{Eu}^{3+}$ Azo-CPAE-2, it was found that the peak at $1672 \mathrm{~cm}^{-1}$ corresponding to the carbonyl groups of DMF was absent in the IR spectrum of $\mathrm{Eu}^{3+}$-Azo-CPAE-2. Alternatively, a new peak at $1663 \mathrm{~cm}^{-1}$ could be observed in the IR spectrum of $\mathrm{Eu}^{3+}$-AzoCPAE-2, which demonstrated that there was some interaction between the carbonyl groups of DMF and $\mathrm{Eu}^{3+}$ ion, suggesting

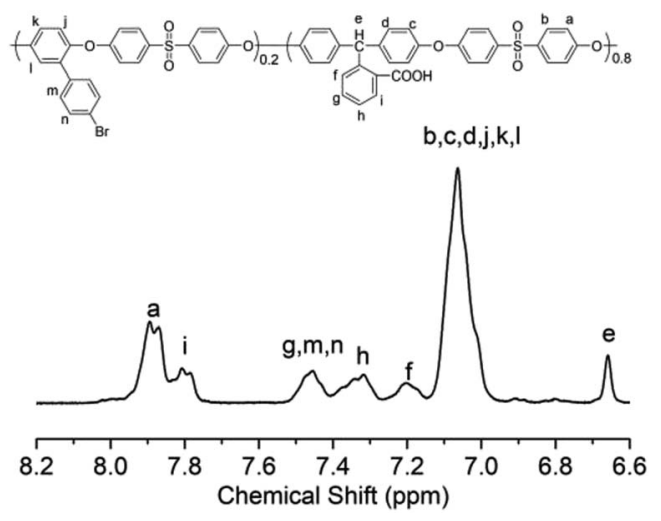

Fig. $3{ }^{1} \mathrm{H}$ NMR spectrum of Br-CPAE in DMSO- $d_{6}$. 


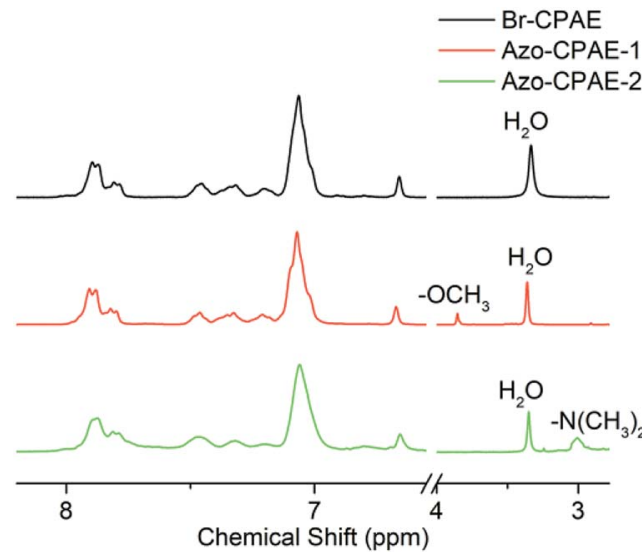

Fig. $4{ }^{1} \mathrm{H}$ NMR spectra of the polymers in DMSO- $d_{6}$.

that the solvent DMF was also involved in the coordination with $\mathrm{Eu}^{3+}$ ion. From the comparison of the IR spectra of Phen and $\mathrm{Eu}^{3+}$-Azo-CPAE-2, it was revealed that the bent vibrations of Phen at $839 \mathrm{~cm}^{-1}$ and $730 \mathrm{~cm}^{-1}$ were redshifted to $819 \mathrm{~cm}^{-1}$ and $727 \mathrm{~cm}^{-1}$, respectively, indicating the formation of coordination bonds between $\mathrm{Eu}^{3+}$ ion and the nitrogen atom of Phen. Moreover, from the comparison of the IR spectra of AzoCPAE-2 and $\mathrm{Eu}^{3+}$-Azo-CPAE-2, it was found that the peak at $1718 \mathrm{~cm}^{-1}$ corresponding to carboxyl groups was absent in the IR spectrum of $\mathrm{Eu}^{3+}$-Azo-CPAE-2. Alternatively, the characteristic absorption bond corresponding to the symmetric stretching vibration of $\mathrm{COO}^{-}$at $1385 \mathrm{~cm}^{-1}$ could be observed in the IR spectrum of $\mathrm{Eu}^{3+}$-Azo-CPAE-2. Accordingly, it could be included that all of Azo-CPAE-2, DMF and Phen participated in the coordination reaction with $\mathrm{Eu}^{3+}$ ions. The chemical structures of other rare earth coordination polymers could also be confirmed by IR. The WAXD patterns of $\mathrm{Eu}^{3+}$-Phen, polymers and rare earth coordination polymers are illustrated in Fig. 7. The WAXD patterns of the polymers displayed an amorphous characteristic, and $\mathrm{Eu}^{3+}$-Phen showed strong diffraction peaks, indicating a crystal lattice structure. However, the WAXD pattern of the rare earth coordination polymers only showed an amorphous structure, which indicated that the rare earth ions were not simply doped into the polymers but coordinated with

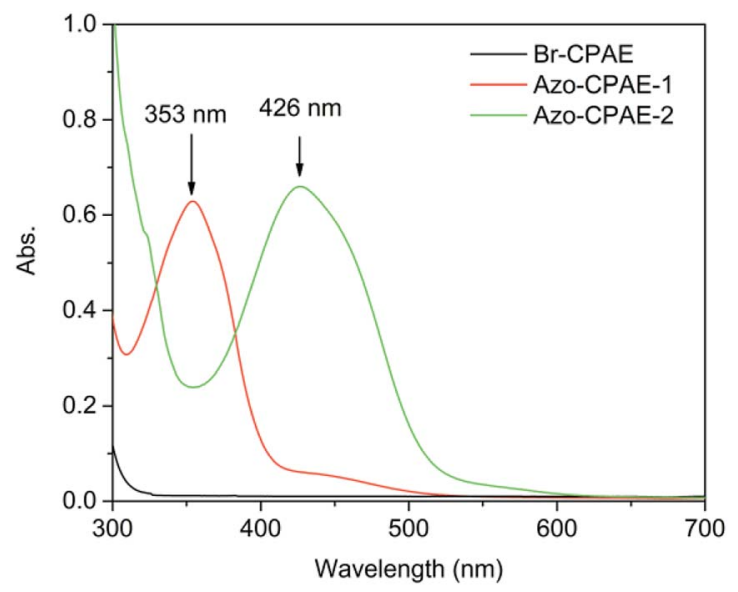

Fig. 5 UV-vis spectra of polymers in DMF solution.



Fig. 6 IR spectra of DMF, Phen, Azo-CPAE-2, and Eu ${ }^{3+}-\mathrm{AzO}-\mathrm{CPAE}-2$.

the carboxyl groups in the side chain of the polymers, leading to a homogeneous distribution of rare earth ions within the polymer host. The WAXD finding was well consistent with the IR analysis results. As demonstrated in other similar polymer systems, ${ }^{22,24,25}$ the rare earth coordination polymer might own a stable eight-fold coordination structure. The possible molecular formula of the rare earth coordination polymer is depicted in Scheme 5 , which could also be confirmed by elemental analysis. As shown in Table 3, the measured values of C, H, N and $\mathrm{Eu}^{3+}$ agreed well with the calculated values.

\subsection{Thermal properties of the polymers}

DSC and TGA measurements were carried out to investigate the thermal properties of the polymers. Fig. 8 shows the DSC curves of the polymers and the experiment data are listed in Table 4. All the polymers showed glass transition temperatures above $218^{\circ} \mathrm{C}$, indicating their high glass transition temperatures. The TGA curves and data of the polymers under nitrogen are shown

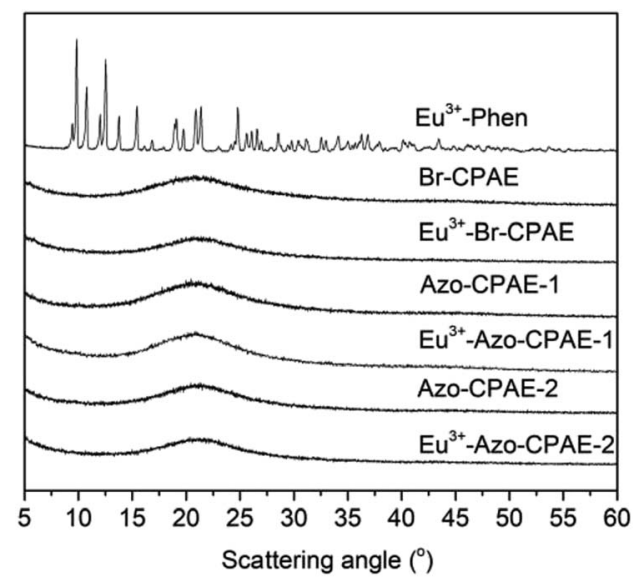

Fig. 7 The WAXD patterns of $\mathrm{Eu}^{3+}-$ Phen, polymers and rare earth coordination polymers. 
in Fig. 9 and Table 3, respectively. The temperatures at 5\% weight loss $\left(T_{\mathrm{d} 5}\right)$ of the polymers were all above $381{ }^{\circ} \mathrm{C}$, indicating their excellent thermal stability. Because the thermal stability of azobenzene chromophores was weaker than the thermal stability of the main chain of Br-CPAE, the temperatures at 5\% weight loss of Azo-CPAE-1 and Azo-CPAE-2 were lower than that of Br-CPAE.

\subsection{Analysis of fluorescence properties}

The excitation and emission spectra of $\mathrm{Eu}^{3+}$-Br-CPAE are shown in Fig. 10. The excitation spectrum of $\mathrm{Eu}^{3+}$-Br-CPAE was obtained by monitoring the emission wavelength at $619 \mathrm{~nm}$. The strongest excitation peak corresponding to $\mathrm{Eu}^{3+}-\mathrm{Br}-\mathrm{CPAE}$ appeared at $339 \mathrm{~nm}$. The emission spectrum of $\mathrm{Eu}^{3+}$-Br-CPAE exhibited the characteristic emission of $\mathrm{Eu}^{3+}$ ion under the excitation at $339 \mathrm{~nm}$. The emission peaks at $580 \mathrm{~nm}, 593 \mathrm{~nm}$, $619 \mathrm{~nm}, 651 \mathrm{~nm}$ and $700 \mathrm{~nm}$ were assigned to the characteristic emission ${ }^{5} \mathrm{D}_{0}{ }^{-7} \mathrm{~F}_{J}(J=0,1,2,3,4)$ transitions of $\mathrm{Eu}^{3+}$ ion, respectively.

\subsection{The influence of absorption wavelength of azobenzene chromophores on the fluorescence properties}

Fig. 11 shows the fluorescence spectra of the rare earth coordination polymers in DMF with the same concentration. All the

Table 3 Elemental analysis of the rare earth complexes

\begin{tabular}{llrr}
\hline Complexes & Element & Found & Calculated \\
\hline $\mathrm{Eu}^{3+}$-Br-CPAE & $\mathrm{C}$ & 63.34 & 62.19 \\
& $\mathrm{H}$ & 4.07 & 4.21 \\
& $\mathrm{~N}$ & 6.53 & 6.40 \\
$\mathrm{Eu}^{3+}$-Azo-CPAE-1 & $\mathrm{Eu}^{3+}$ & 11.25 & 11.66 \\
& $\mathrm{C}$ & 65.01 & 63.24 \\
& $\mathrm{H}$ & 4.13 & 4.29 \\
$\mathrm{Eu}^{3+}$-Azo-CPAE-2 & $\mathrm{N}$ & 6.27 & 6.70 \\
& $\mathrm{Eu}$ & 11.53 & 11.37 \\
& $\mathrm{C}$ & 64.72 & 63.70 \\
& $\mathrm{H}$ & 4.27 & 4.34 \\
& $\mathrm{~N}$ & 7.05 & 6.94 \\
& $\mathrm{Eu}^{3+}$ & 10.98 & 11.32
\end{tabular}

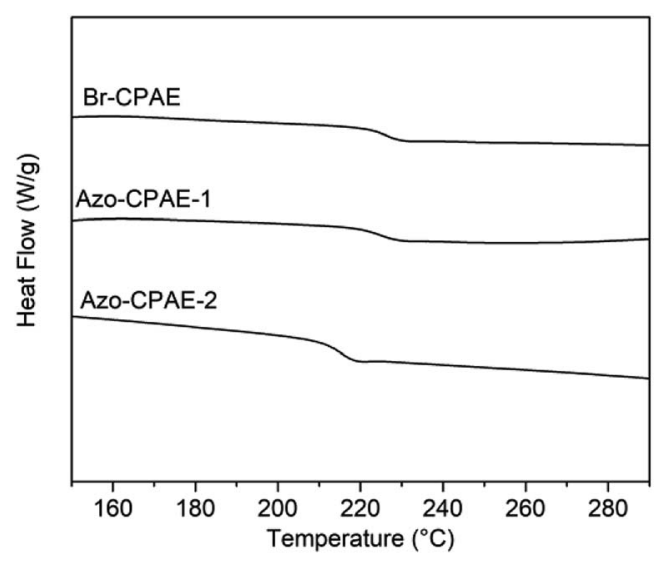

Fig. 8 DSC curves of the polymers in nitrogen.
Table 4 Thermal properties of the polymers

\begin{tabular}{llll}
\hline Polymer & $T_{\mathrm{g}}{ }^{a}\left({ }^{\circ} \mathrm{C}\right)$ & $T_{\mathrm{d} 5}{ }^{b}\left({ }^{\circ} \mathrm{C}\right)$ & $T_{\mathrm{d} 10}{ }^{c}\left({ }^{\circ} \mathrm{C}\right)$ \\
\hline Br-CPAE & 231 & 413 & 430 \\
Azo-CPAE-1 & 227 & 387 & 423 \\
Azo-CPAE-2 & 218 & 381 & 412
\end{tabular}

${ }^{a}$ Glass transition temperature by DSC. ${ }^{b} 5 \%$ weight-loss temperatures were detected at a heating rate of $10{ }^{\circ} \mathrm{C} \mathrm{min}{ }^{-1}$ in nitrogen. ${ }^{c} 10 \%$ weight-loss temperatures were detected at a heating rate of $10{ }^{\circ} \mathrm{C} \mathrm{m^{-1 }}$ in nitrogen.

rare earth coordination polymers were prepared from polymers, $\mathrm{Eu}^{3+}$ ion and Phen at a molar ratio of $1: 0.8: 2.4$. It could be observed that the fluorescent intensity of $\mathrm{Eu}^{3+}$-Azo-CPAE-2 was much larger than that of $\mathrm{Eu}^{3+}$-Azo-CPAE-1, which was close to the fluorescent intensity of $\mathrm{Eu}^{3+}$-Br-CPAE. The phenomenon could be interpreted as follows. The excitation wavelengths of $\mathrm{Eu}^{3+}$ ion overlapped with the absorption wavelength of azobenzene chromophores in $\mathrm{Eu}^{3+}$-Azo-CPAE-1, so the excitation light of rare earth complexes would be absorbed by the azobenzene chromophores, which induced the energy competition between azobenzene chromophores and $\mathrm{Eu}^{3+}$ ion. ${ }^{26,27}$ On the other hand, the absorption wavelength of azobenzene

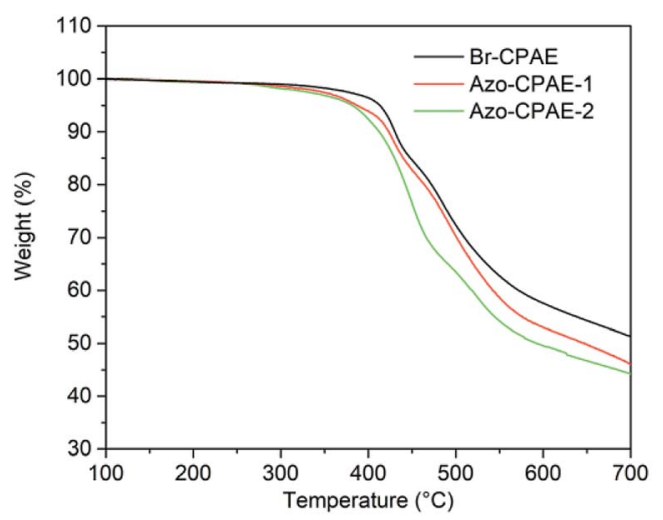

Fig. 9 TGA curves of the polymers in nitrogen.

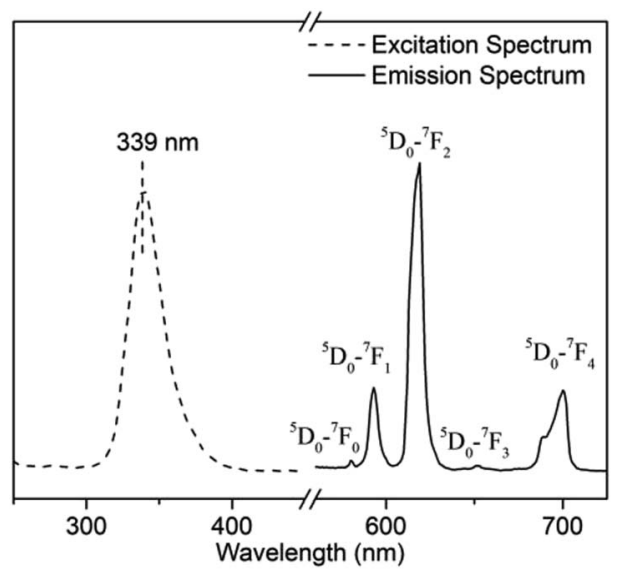

Fig. 10 Excitation and emission spectra of $\mathrm{Eu}^{3+}-\mathrm{Br}-\mathrm{CPAE}$. 


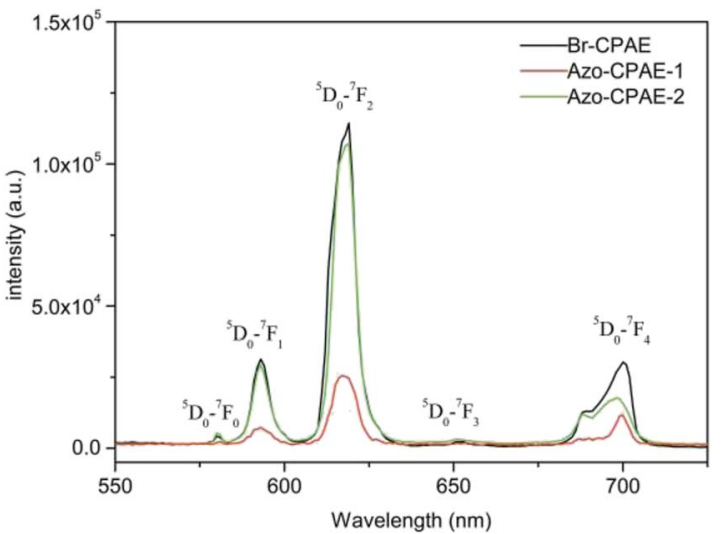

Fig. 11 Emission spectra of the rare earth coordination polymers in DMF.

chromophores in $\mathrm{Eu}^{3+}$-Azo-CPAE-2 was far away from the excitation wavelengths of $\mathrm{Eu}^{3+}$ ion, so the luminescent efficiency of the rare earth complexes would not be reduced significantly.

\subsection{Photoinduced surface-relief-gratings of $\mathrm{Eu}^{3+}$-Azo-CPAE-2}

Based on the results of the fluorescence properties of the rare earth coordination polymers, $\mathrm{Eu}^{3+}$-Azo-CPAE-2 was a much better material than $\mathrm{Eu}^{3+}$-Azo-CPAE-1 to prepare fluorescent holographic micropatterns. To fabricate SRGs, the film of $\mathrm{Eu}^{3+}$ Azo-CPAE-2 was exposed to two polarized interfering laser beams for $30 \mathrm{~s}$, and SRGs were rapidly formed on the films. Fig. 12 shows a typical AFM plane image of the SRGs formed on the $\mathrm{Eu}^{3+}$-Azo-CPAE-2 film. AFM section analysis revealed that the modulation depth and the grating spacing of the SRGs of $\mathrm{Eu}^{3+}$-Azo-CPAE-2 were $40 \mathrm{~nm}$ and $256 \mathrm{~nm}$, respectively. Compared to the other SRGs we reported before, ${ }^{19}$ the modulation depth of the SRGs was shallower under similar
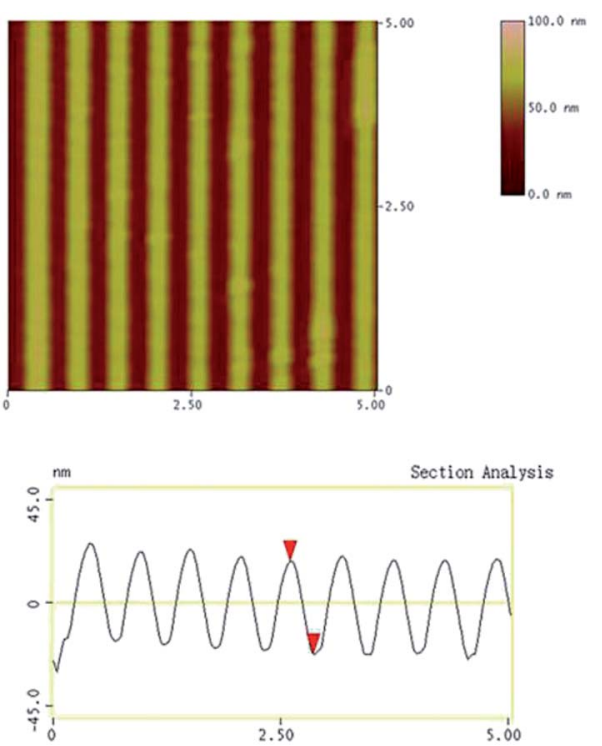

Fig. 12 AFM plane view and section analysis of the SRGs formed on $\mathrm{Eu}^{3+}-\mathrm{AzO}-\mathrm{CPAE}-2$ film.



Fig. 13 Fluorescence microscopy image of SRGs on $\mathrm{Eu}^{3+}$-Azo-CPAE2 film.

experimental conditions, due to that the absorption bands of Azo-CPAE-2 did not match the wavelength of the laser beams (355 nm) well. However, the modulation depth could be adjusted by the irradiation time and the irradiation energy, and the grating spacing could be controlled by adjusting the angle between the two interfering beams. ${ }^{1}$ Fig. 13 presents the fluorescence microscopy image of SRGs on the $\mathrm{Eu}^{3+}$-Azo-CPAE-2 film. Under the excitation, the regular patterning obtained on the $\mathrm{Eu}^{3+}$-Azo-CPAE-2 film exhibited red light.

\section{Conclusions}

In this work, two novel azobenzene-containing poly(aryl ether)s with different absorption wavelengths were synthesized via Ullmann coupling and Sonogashira coupling, respectively. Their structures were characterized by IR, ${ }^{1} \mathrm{H}$ NMR and UV-vis. These polymers exhibited a high glass transition temperature $\left(T_{\mathrm{g}}>218^{\circ} \mathrm{C}\right)$ and good thermal stability $\left(T_{\mathrm{d} 5}>381{ }^{\circ} \mathrm{C}\right)$. Rare earth complexes were prepared by using the two novel azobenzene-containing poly(aryl ether)s as macromolecular ligands. The rare earth complexes based on Azo-CPAE- 2 whose absorption wavelength was far from the excitation wavelengths of the rare earth complexes showed a much larger fluorescence intensity than the rare earth complexes based on Azo-CPAE-1. By exposing the film of the rare earth complexes based on Azo-CPAE-2 to two interference laser beams, SRGs can be formed on the film and can be detected by fluorescence microscopy measurement.

\section{Conflicts of interest}

There are no conflicts to declare.

\section{Acknowledgements}

The authors gratefully acknowledge the Liaoning Province Doctor Startup Fund (Grant No. 201501130) and the National Natural Science Foundation of China (Grant No. 51703089) for financial support. 


\section{References}

1 A. Natansohn and P. Rochon, Chem. Rev., 2002, 102, 41394175.

2 A. S. Matharu, S. Jeeva, P. R. Huddleston and P. S. Ramanujam, J. Mater. Chem., 2007, 17, 4477-4482.

3 C. Appiah, K. R. Siefermann, M. Jorewitz, H. Barqawi and W. H. Binder, RSC Adv., 2016, 6, 6358-6367.

4 T. Ikeda, J. I. Mamiya and Y. Yu, Angew. Chem., Int. Ed., 2007, 46, 506-528.

5 Y. L. Yu, M. Nakano and T. Ikeda, Nature, 2003, 425, 145.

6 H. Y. Wen, W. D. Zhang, Y. Y. Wengm and Z. J. Hu, RSC Adv., 2014, 4, 11776-11781.

7 N. J. Li, J. M. Lu, H. Li and E. T. Kang, Dyes Pigm., 2011, 80, 18-24.

8 X. B. Chen, J. J. Zhang, H. B. Zhang, Z. H. Jiang ZH, G. Shi and Y. L. Song, Dyes Pigm., 2008, 77, 223-228.

9 P. Rochon, E. Batalla and A. Natansohn, Appl. Phys. Lett., 1995, 66, 136-138.

10 D. Y. Kim, S. K. Tripathy, L. Li and J. Kumar, Appl. Phys. Lett., 1995, 66, 1166-1168.

11 I. Sava, A. Burescu, I. Stoica, V. Musteata, M. Cristea, I. Mihaila, V. Pohoata and I. Topala, $R S C A d v ., 2015,5$, 10125-10133.

12 J. J. Zhang, H. B. Zhang, X. B. Chen, J. H. Pang, Y. X. Zhang, Y. P. Wang, Q. D. Chen, S. H. Pei, W. X. Peng and Z. H. Jiang, React. Funct. Polym., 2011, 71, 553-560.

13 Y. X. Zhang, S. H. Pei, Y. P. Wang, Z. D. Cui, N. Li, Y. Zhu, H. B. Zhang and Z. H. Jiang, Dyes Pigm., 2013, 99, 1117-1123.

14 X. B. Chen, Y. H. Zhang, B. J. Liu, J. J. Zhang, H. Wang, W. Y. Zhang, Q. D. Chen, S. H. Pei and Z. H. Jiang, J. Mater. Chem., 2008, 18, 5019-5026.
15 J. J. Zhang, H. B. Zhang, X. B. Chen, Y. H. Zhang, X. F. Li, Q. D. Chen and Z. H. Jiang, Mater. Lett., 2010, 64, 337-340.

16 E. Ishow, A. Brosseau, G. Clavier, K. Nakatani, R. Pansu, J. J. Vachon, P. Tauc, D. Chauvat, C. Mendonca and E. Piovesan, J. Am. Chem. Soc., 2007, 129, 8970-8971.

17 L. H. Liu, K. Nakatani, R. Pansu, J. J. Vachon, P. Tauc and E. Ishow, Adv. Mater., 2007, 19, 433-436.

18 X. B. Chen, B. J. Liu, H. B. Zhan, S. W. Guan, J. J. Zhang, Z. H. Jiang and M. D. Guiver, Langmuir, 2009, 25, 1044410446.

19 Y. X. Zhang, J. J. Zhang, Z. D. Cui, Q. D. Chen, H. B. Zhang and Z. H. Jiang, J. Polym. Sci., Part A: Polym. Chem., 2015, 53, 936-943.

20 S. F. Xiao, X. M. Lu, A. H. Lu and B. Su, Macromolecules, 2008, 41, 3884-3892.

21 X. Y. Ma, Z. H. Lv, D. Wang, S. W. Guan, C. H. Chen, G. B. Wang, D. M. Zhang and Z. H. Jian, J. Photochem. Photobiol., A, 2007, 188, 43-50.

22 D. Liu and Z. G. Wang, Polymer, 2008, 49, 4960-4967.

23 H. M. D. Bandara and S. C. Burdette, Chem. Soc. Rev., 2012, 41, 1809-1825.

24 J. Z. Jiang, Y. Z. Bian, F. Furuya, W. Liu, M. T. M. Choi and N. Kobayashi, Chem.-Eur. J., 2001, 7, 5059-5069.

25 D. Liu, H. Yu, Z. G. Wang and Q. P. Nie, Polym. Int., 2010, 59, 937-944.

26 D. Wang, J. Y. Zhang, H. Ming, Q. Yan, Q. J. Zhang, P. Wang, J. Yang and Z. Q. Zheng, Chin. Phys. Lett., 2004, 21, 24452447.

27 Q. Yan, S. Wu, Y. L. Chen, Y. H. Luo and Q. J. Zhang, Spectrochim. Acta, Part A, 2009, 71, 1644-1647. 